\title{
INFINITE TOWERS OF TREE LATTICES
}

\author{
Lisa Carbone and Gabriel RosenberG
}

\section{Introduction}

Let $X$ be a locally finite tree and let $G=\operatorname{Aut}(X)$. Then $G$ is naturally a locally compact group ([BL], Ch.3). A discrete subgroup $\Gamma \leq G$ is called an $X$-lattice if

$$
\operatorname{Vol}(\Gamma \backslash \backslash X) \quad:=\sum_{x \in V(\Gamma \backslash X)} \frac{1}{\left|\Gamma_{x}\right|}
$$

is finite, and a uniform $X$-lattice if $\Gamma \backslash X$ is a finite graph, non-uniform otherwise ([BL], Ch.3). Bass and Kulkarni have shown ([BK], (4.10)) that $G=\operatorname{Aut}(X)$ contains a uniform $X$-lattice if and only if $X$ is the universal covering of a finite connected graph, or equivalently, that $G$ is unimodular and $G \backslash X$ is finite. In this case, we call $X$ a uniform tree.

Following ([BL], (3.5)) we call $X$ rigid if $G$ itself is discrete, and we call $X$ minimal if $G$ acts minimally on $X$, that is, there is no proper $G$-invariant subtree. If $X$ is uniform then there is always a unique minimal $G$-invariant subtree $X_{0} \subseteq X$ ([BL] (5.7), (5.11), (9.7)). We call $X$ virtually rigid if $X_{0}$ is rigid $(c f .([\mathrm{BL}],(3.6))$.

Let $X$ be a locally finite tree, and let $\Gamma \leq \Gamma^{\prime}$ be an inclusion of $X$-lattices. Then by ([BL], (1.7)) we have:

$$
\operatorname{Vol}\left(\Gamma^{\prime} \backslash \backslash X\right)=\frac{\operatorname{Vol}(\Gamma \backslash \backslash X)}{\left[\Gamma^{\prime}: \Gamma\right]} .
$$

We call an infinite ascending chain

$$
\Gamma_{1}<\Gamma_{2}<\Gamma_{3}<\ldots
$$

of $X$-lattices an infinite tower of $X$-lattices. By (0.2), the lattice inclusions of (0.3) are of finite index, and $\operatorname{Vol}\left(\Gamma_{i} \backslash \backslash X\right) \longrightarrow 0$ as $i \longrightarrow \infty$.

The Kazhdan-Margulis property for lattices in Lie groups $([\mathrm{KM}])$ states that the covolume of a lattice is bounded away from zero. Hence the existence of

\footnotetext{
Received November 16, 2000

The first author was supported in part by NSF grant DMS-9800604, and the second by NSF grant \#DMS-9810750.

2000 Mathematics Subject Classification. Primary 20F32; Secondary 22F50.
} 
infinite towers of $X$-lattices in $G=\operatorname{Aut}(X)$ shows that the Kazhdan-Margulis property is violated for $X$-lattices.

Bass and Kulkarni have given ([BK], (Sec.7)) several examples of uniform trees such that $G=\operatorname{Aut}(X)$ contains infinite towers of uniform $X$-lattices. The second author has extended the results and techniques of Bass-Kulkarni to all uniform trees that are not rigid $([\mathrm{R}])$.

Here our main result is that, with one exception (see $\S 5$ ), if $G=\operatorname{Aut}(X)$ contains a non-uniform $X$-lattice, then $G$ contains an infinite tower of nonuniform $X$-lattices.

The authors would like to thank H. Bass for many helpful discussions and suggestions.

\section{The setting}

An edge-indexed graph $(A, i)$ consists of an underlying graph $A$, and an assignment of a positive integer $i(e)>0$ to each oriented edge $e \in E A$. Our underlying graph $A$ will always be understood to be locally finite. In $[\mathrm{BK}]$ and [BL] one allows $i(e)$ to be any positive cardinal, but our interest here is only in finite $i(e)$. If $i(e)>1$, we call $e$ ramified and unramified otherwise.

Let $\mathbb{A}=(A, \mathcal{A})$ be a graph of groups, with underlying graph $A$, vertex groups $\left(\mathcal{A}_{a}\right)_{a \in V A}$, edge groups $\left(\mathcal{A}_{e}=\mathcal{A}_{\bar{e}}\right)_{e \in E A}$ and monomorphisms $\alpha_{e}: \mathcal{A}_{e} \hookrightarrow \mathcal{A}_{\partial_{0} e}$. A graph of groups $\mathbb{A}$ naturally gives rise to an edge-indexed graph $I(\mathbb{A})=(A, i)$ whose indices are the indices of the edge groups as subgroups of the adjacent vertex groups: that is, $i(e)=\left[\mathcal{A}_{\partial_{0} e}: \alpha_{e} \mathcal{A}_{e}\right]$, which we assume to be finite, for all $e \in E A$.

Given an edge-indexed graph $(A, i)$, a graph of groups $\mathbb{A}$ such that $I(\mathbb{A})=$ $(A, i)$ is called a grouping of $(A, i)$. We call $\mathbb{A}$ a finite grouping if the vertex groups $\mathcal{A}_{a}$ are finite and a faithful grouping if $\mathbb{A}$ is a faithful graph of groups, that is, if $\pi_{1}(\mathbb{A}, a)$ acts faithfully on $X=\widetilde{(\mathbb{A}, a)}$.

Let $\mathbb{A}^{\prime}$ and $\mathbb{A}$ be groupings of $(A, i)$. Then $\mathbb{A}^{\prime}=\left(A, \mathcal{A}^{\prime}\right)$ is called a full graph of subgroups of $\mathbb{A}=(A, \mathcal{A})$ (as in ([B], (1.14)) if $\mathcal{A}_{a}^{\prime} \leq \mathcal{A}_{a}$ for $a \in A^{\prime}$, and for $e \in E A^{\prime}, \mathcal{A}_{e}^{\prime} \leq \mathcal{A}_{e}$, and $\alpha_{e}^{\prime}=\left.\alpha_{e}\right|_{\mathcal{A}_{e}^{\prime}}$. We further assume that for $e \in E A^{\prime}$, with $\partial_{0} e=a, \mathcal{A}_{a}^{\prime} \cap \alpha_{e} \mathcal{A}_{e}=\alpha_{e} \mathcal{A}_{e}^{\prime}$, that is $\mathcal{A}_{a}^{\prime} / \alpha_{e} \mathcal{A}_{e}^{\prime} \longrightarrow \mathcal{A}_{a} / \alpha_{e} \mathcal{A}_{e}$ is injective, and hence bijective. This assumption implies that $I\left(\mathbb{A}^{\prime}\right)=(A, i)$, and that $\pi_{1}\left(\mathbb{A}^{\prime}, a^{\prime}\right) \leq \pi_{1}(\mathbb{A}, a)([\mathrm{B}],(1.14))$.

Let $(A, i)$ be an edge-indexed graph. A tower of groupings on $(A, i)$ is a semiinfinite sequence $\left(\mathbb{A}_{i}\right)_{i \in \mathbb{Z}_{>0}}$ of groupings of $(A, i)$ such that each $\mathbb{A}_{i}$ is a full graph of proper subgroups of $\mathbb{A}_{i+1}$. A tower of faithful groupings induces an infinite ascending chain of fundamental groups:

$$
\pi_{1}\left(\mathbb{A}_{1}, a_{0}\right) \leq \pi_{1}\left(\mathbb{A}_{2}, a_{0}\right) \leq \pi_{1}\left(\mathbb{A}_{3}, a_{0}\right) \leq \ldots
$$

For an edge $e \in E A$, define:

$$
\Delta(e) \quad:=\frac{i(\bar{e})}{i(e)}
$$


If $\gamma=\left(e_{1}, \ldots, e_{n}\right)$ is a path, set:

$$
\Delta(\gamma):=\Delta\left(e_{1}\right) \ldots \Delta\left(e_{n}\right) .
$$

Definition. An edge-indexed graph $(A, i)$ is called unimodular if $\Delta(\gamma)=1$ for all closed paths $\gamma$ in $A$.

Now assume that $(A, i)$ is unimodular. Pick a base point $a_{0} \in V A$, and define, for $a \in V A$,

$$
N_{a_{0}}(a):=\frac{\Delta a}{\Delta a_{0}}\left(=\Delta(\gamma) \text { for any path } \gamma \text { from } a_{0} \text { to } a\right) \in \mathbb{Q}_{>0} .
$$

For $e \in E A$, put

$$
N_{a_{0}}(e) \quad:=\frac{N_{a_{0}}\left(\partial_{0}(e)\right)}{i(e)} .
$$

Following $([\mathrm{BL}],(2.6))$, we say that $(A, i)$ has bounded denominators if

$$
\left\{N_{a_{0}}(e) \mid e \in E A\right\}
$$

has bounded denominators, that is, if for some integer $D>0, D \cdot N_{a_{0}}$ takes only integer values on edges. Since

$$
N_{a_{1}}=\frac{\Delta a_{0}}{\Delta a_{1}} N_{a_{0}}
$$

this condition is independant of $a_{0} \in V A$.

Theorem ([BK], (2.4)). An indexed graph $(A, i)$ admits a finite grouping if and only if $(A, i)$ is unimodular and has bounded denominators. The grouping can further be taken to be faithful.

As in ([BL], Ch.2) we define the volume of an indexed graph $(A, i)$ at a basepoint $a_{0} \in V A$ :

$$
\operatorname{Vol}_{a_{0}}(A, i):=\sum_{a \in V A} \frac{1}{\left(\frac{\Delta a}{\Delta a_{0}}\right)}=\sum_{a \in V A}\left(\frac{\Delta a_{0}}{\Delta a}\right) .
$$

Then

$$
\operatorname{Vol}_{a_{1}}(A, i)=\frac{\Delta a_{0}}{\Delta a_{1}} \operatorname{Vol}_{a_{0}}(A, i),
$$

([BL], Ch. 2) We write $\operatorname{Vol}(A, i)<\infty$ if $\operatorname{Vol}_{a}(A, i)<\infty$ for some, and hence every $a \in V A$.

If $\mathbb{A}$ is a finite grouping of $(A, i)$, then we have ([BL], (2.6.15)):

$$
\operatorname{Vol}(\mathbb{A})=\frac{1}{\left|\mathcal{A}_{a}\right|} \operatorname{Vol}_{a}(A, i)
$$


which is automatically finite if $\operatorname{Vol}(A, i)<\infty$.

We now describe a method for constructing $X$-lattices which follows naturally from the fundamental theory of Bass-Serre ([B], $[\mathrm{S}])$, and was first suggested in $([\mathrm{BK}])$. We begin with an edge-indexed graph $(A, i)$. Then $(A, i)$ determines $X=\left(\widetilde{A, i, a_{0}}\right)$ up to isomorphism ([BL], Ch. 2$)$.

We say that $(A, i)$ admits a lattice if $(A, i)$ admits a grouping $\mathbb{A}$ such that $\pi_{1}\left(\mathbb{A}, a_{0}\right)$ is an $X$-lattice. This happens if and only if $(A, i)$ satisfies:

(U) $(A, i)$ is unimodular, and

(BD) $(A, i)$ has bounded denominators, and

(FV) $(A, i)$ has finite volume.

Assume that $(A, i)$ is unimodular and has bounded denominators (which is automatic if $A$ if finite). By ([BK], (2.4)) we can find a finite faithful grouping $\mathbb{A}$ of $(A, i)$ and a group $\Gamma=\pi_{1}\left(\mathbb{A}, a_{0}\right)$ acting faithfully on $X$. Then

(a) $\Gamma$ is discrete, since $\mathbb{A}$ is a graph of finite groups.

(b) $\Gamma$ is a uniform $X$-lattice if and only if $A$ is finite.

(c) $\Gamma$ is a non-uniform $X$-lattice if and only if $A$ is infinite, and

$$
\operatorname{Vol}(\Gamma \backslash \backslash X)=\operatorname{Vol}(\mathbb{A})\left(:=\sum_{a \in V A} \frac{1}{\left|\mathcal{A}_{a}\right|}=\frac{1}{\left|\mathcal{A}_{a}\right|} \operatorname{Vol}_{a}(A, i)\right)<\infty .
$$

Our task is the following: given an edge-indexed graph $(A, i)$ of finite volume, construct an infinite tower of finite faithful groupings of $(A, i)$. This induces an infinite tower

$$
\Gamma_{1}<\Gamma_{2}<\Gamma_{3}<\ldots
$$

of $X$-lattices in $\operatorname{Aut}(X)$, for $X=\widetilde{(A, i)}$, with $\Gamma_{i} \backslash X=A, i=1,2, \ldots$.

An edge $e \in E A$ is called separating if $A-\{e, \bar{e}\}$ has two connected components $A_{0}(e)$ and $A_{1}(e)$, where $A_{0}(e)$ and $A_{1}(e)$ contain $\partial_{0}(e)$ and $\partial_{1}(e)$ respectively.

Let $(A, i)$ be any connected edge-indexed graph. A subset $\beta \subset E A$ of $n \geq 2$ (oriented) edges is called an arithmetic bridge for $(A, i)$ (as in $([\mathrm{C} 1]$, Sec. 4$)$ ) if:

(1) $\beta \cap \bar{\beta}=\varnothing, A-(\beta \cup \bar{\beta})$ has two connected components, $A_{0}$ and $A_{1}$,

(2) For every $e \in \beta, \partial_{0} e \in A_{0}$ and $\partial_{1} e \in A_{1}$,

(3) There exists an integer $d>1$ such that $d \mid i(e)$ for every $e \in \beta$.

Following $[\mathrm{BT}]$ we say that $(A, i)$ is discretely ramified if for $e \in E A$

$i(e)>1 \Longrightarrow i(e)=2, e$ is separating, and $\left(A_{0}(e), i\right)$ is an unramified tree.

We call $(A, i)$ a dominant-rooted edge-indexed tree if $A$ is a tree and if there exists an $a \in V A$ such that $i(e)=1$ for all edges $e \in E A$ directed towards $a$. Let $(A, i)$ be an edge-indexed graph. We say that $(A, i)$ is restricted if $(A, i)$ satisfies any one of the following conditions:

(DR) $(A, i)$ is discretely ramified, or

(F) $(A, i)$ is a dominant-rooted edge-indexed tree, or

(GS) $A$ is a tree, and $(A, i)$ contains a prime-prime interval (see $[\mathrm{R}]$ ) and no other ramified edges. 
We say that $(A, i)$ is permissible if $(A, i)$ admits a lattice and if $(A, i)$ is not restricted. We note that an infinite edge-indexed graph $(A, i)$ with finite volume is automatically non-discretely ramified, is not a dominant-rooted tree ([CR2]), is obviously not (GS) as above, and hence is not restricted.

\section{Rooted products of graphs of groups}

Given rooted graphs of groups $\mathbb{A}=\left(A, \mathcal{A}, a_{0}\right), a_{0} \in V A$, and $\mathbb{B}=\left(B, \mathcal{B}, b_{0}\right)$, $b_{0} \in V B$, we construct a rooted graph of groups $\mathbb{C}=\left(C, \mathcal{C}, c_{0}\right)=\mathbb{A} \times \times_{a_{0}=b_{0}} \mathbb{B}$ as follows: we set

$$
C:=A \sqcup B /\left(a_{0}=b_{0}=c_{0}\right) .
$$

For $a \in V A, e \in E A$, we set

$$
\mathcal{C}_{a}:=\mathcal{A}_{a} \times \mathcal{B}_{b_{0}}, \quad \mathcal{C}_{e}:=\mathcal{A}_{e} \times \mathcal{B}_{b_{0}},
$$

and if $\alpha_{e}: \mathcal{A}_{e} \hookrightarrow \mathcal{A}_{\partial_{0} e}$, we set

$$
\gamma_{e}:=\alpha_{e} \times I d_{B_{b_{0}}} .
$$

Similarly, for $b \in V B, e \in E B$, we set

$$
\mathcal{C}_{b}:=\mathcal{A}_{a_{0}} \times \mathcal{B}_{b}, \quad \mathcal{C}_{e}:=\mathcal{A}_{a_{0}} \times \mathcal{B}_{e},
$$

and if $\beta_{e}: \mathcal{B}_{e} \hookrightarrow \mathcal{B}_{\partial_{0} e}$, we set

$$
\gamma_{e}:=I d_{A_{a_{0}}} \times \beta_{e}
$$

If we set $\left(A, i^{A}\right)=I(\mathbb{A})$ and $\left(B, i^{B}\right)=I(\mathbb{B})$, then we have the 'rooted union of edge-indexed graphs':

$$
\left(C, i^{C}\right) \quad:=\left(A, i^{A}\right) \sqcup\left(B, i^{B}\right) /\left(a_{0}=b_{0}=c_{0}\right),
$$

for $c_{0} \in V C$, and clearly $\mathbb{C}$ is a grouping of $\left(C, i^{C}\right)$.

\section{(2.1) Remarks.}

(1) The graph of groups $\mathbb{C}$ is faithful if and only if $\mathbb{A}$ and $\mathbb{B}$ are faithful. In fact, if $N_{\mathbb{A}}$ is the maximal normal subgroup of $\mathbb{A}$, and $N_{\mathbb{B}}$ is the maximal normal subgroup of $\mathbb{B}$, then the maximal normal subgroup of $\mathbb{C}$ is $N_{\mathbb{A}} \times N_{\mathbb{B}}$.

(2) We have

$$
\pi_{1}\left(\mathbb{C}, c_{0}\right)=\left(\pi_{1}\left(\mathbb{A}, a_{0}\right) \times \mathcal{B}_{b_{0}}\right) \quad *_{\left(\mathcal{A}_{a_{0}} \times \mathcal{B}_{b_{0}}\right)} \quad\left(\mathcal{A}_{a_{0}} \times \pi_{1}\left(\mathbb{B}, b_{0}\right)\right) .
$$

(3) If $\mathbb{A}$ and $\mathbb{B}$ are graphs of finite groups, then so also is $\mathbb{C}$, and

$$
\operatorname{Vol}(\mathbb{C})=\frac{1}{\left|\mathcal{B}_{b_{0}}\right|} \operatorname{Vol}(\mathbb{A})+\frac{1}{\left|\mathcal{A}_{a_{0}}\right|} \operatorname{Vol}(\mathbb{B})-\frac{1}{\left|\mathcal{A}_{a_{0}}\right|\left|\mathcal{B}_{b_{0}}\right|} .
$$




\section{(2.2) Functoriality.}

Suppose that we have groupings $\mathbb{A} \leq \mathbb{A}^{\prime}$ of an edge-indexed graph $\left(A, i^{A}\right)$ and $\mathbb{B} \leq \mathbb{B}^{\prime}$ of an edge-indexed graph $\left(B, i^{B}\right)$, then we get groupings

$$
\mathbb{C}=\mathbb{A} \times_{a_{0}=b_{0}} \mathbb{B} \leq \mathbb{C}^{\prime}=\mathbb{A}^{\prime} \times_{a_{0}=b_{0}} \mathbb{B}^{\prime}
$$

for $a_{0} \in V A, b_{0} \in V B$ of the edge-indexed graph

$$
\left(C, i^{C}\right)=\left(A, i^{A}\right) \sqcup\left(B, i^{B}\right) /\left(a_{0}=b_{0}=c_{0}\right),
$$

for $c_{0} \in V C$. In particular, for an edge $e \in V A$ with initial vertex $a \in V A$,

$$
\begin{array}{lcl}
\mathcal{A}_{e}^{\prime} \times \mathcal{B}_{b_{0}}^{\prime} & \stackrel{\alpha_{e}^{\prime} \times I d_{B_{b_{0}}^{\prime}}}{\longrightarrow} & \mathcal{A}_{a}^{\prime} \times \mathcal{B}_{b_{0}}^{\prime} \\
\leq \quad \leq & \leq & \leq \\
& & \leq \\
\mathcal{A}_{e} \times \mathcal{B}_{b_{0}} & \stackrel{\alpha_{e} \times I d_{B_{b_{0}}}}{\longrightarrow} & \mathcal{A}_{a} \times \mathcal{B}_{b_{0}}
\end{array}
$$

commutes, and similarly in $B$.

(2.3) Corollary. A tower $\mathbb{A}_{1} \leq \mathbb{A}_{2} \leq \mathbb{A}_{3} \leq \ldots$ yields a tower

$$
\mathbb{A}_{1} \times_{a_{0}=b_{0}} \mathbb{B} \leq \mathbb{A}_{2} \times_{a_{0}=b_{0}} \mathbb{B} \leq \mathbb{A}_{3} \times_{a_{0}=b_{0}} \mathbb{B} \leq \ldots
$$

Since a unimodular edge-indexed graph with bounded denominators admits a finite faithful grouping, we can apply the above corollary repeatedly to obtain the following lemma.

(2.4) Lemma. Let $(A, i)$ be an edge-indexed graph and let $\left(A_{0}, i\right)$ be a core subgraph such that $(A, i)$ is obtained from $\left(A_{0}, i\right)$ by attaching to finitely many vertices $a_{1}, \ldots, a_{n} \in V A_{0}$, rooted edge-indexed graphs $\left(A_{j}, i_{j}, a_{j}\right), j=1, \ldots, n$ respectively. Suppose that $\left(A_{0}, i\right)$ admits an infinite ascending chain of finite faithful groupings of finite volume. Suppose that each of the $\left(A_{j}, i_{j}\right), j=1, \ldots, n$ are unimodular, have finite volume and bounded denominators.Then $(A, i)$ admits an infinite tower of finite faithful groupings of finite volume.

\section{Infinite towers of uniform tree lattices}

In $[R]$, the second author proved the following:

(3.1) Theorem $([\mathbf{R}])$. Let $(A, i)$ be a finite permissible edge-indexed graph. Then $(A, i)$ admits an infinite tower of finite faithful groupings.

The proof of Theorem (3.1) generalizes the techniques of Bass-Kulkarni ([BK]) for constructing towers of groupings on certain fundamental examples, and uses certain constructions with edge-indexed graphs to extend to a more general setting.

Theorem (3.1) yields the following: 
(3.2) Theorem $([\mathbf{R}])$. Let $X$ be a locally finite tree. The following conditions are equivalent:

(a) $X$ is uniform and not rigid.

(b) $X$ is the universal cover of a finite permissible edge-indexed graph.

(c) Aut $(X)$ contains an infinite ascending chain

$$
\Gamma_{1}<\Gamma_{2}<\Gamma_{3}<\cdots
$$

of uniform $X$-lattices.

(d) The set of uniform covolumes

$$
\{\operatorname{Vol}(\Gamma \backslash \backslash X) \mid \Gamma \text { is a uniform } X \text {-lattice }\} \subset \mathbb{Q}>0
$$

is not bounded away from zero.

This generalizes Theorem 7.1(a) of [BK] which states the result for homogeneous trees.

\section{Infinite towers of non-uniform $X$-lattices with quotient a tree}

The techniques described in $\S 3$ extend to certain infinite edge-indexed graphs. We have the following:

(4.1) Theorem. Let $(A, i)$ be an edge-indexed graph that admits a lattice, and which is infinite, hence permissible. Assume that $(A, i)$ is a tree, but is not dominant-end-rooted. Then $(A, i)$ admits an infinite tower of finite faithful groupings.

Except for the case that $(A, i)$ is a dominant-end-rooted edge-indexed tree (similar to a dominant-rooted edge-indexed tree as defined on page 4, except that an end takes the role given to the root vertex before, that is, $\mathrm{i}(\mathrm{e})=1$ for all edges directed towards that end as opposed to the root vertex (see [CR2] for more details)), the assumption that $(A, i)$ is an infinite permissible tree implies the existence of a finite permissible 'core' graph $\left(A_{0}, i\right)$ which is an edge-indexed path of length $n \geq 1$. By Theorem (3.1), $\left(A_{0}, i\right)$ admits an infinite tower of finite faithful groupings, and we may then apply Lemma (2.4) to extend the tower of groupings to $(A, i)$.

If $(A, i)$ is a dominant-end-rooted edge-indexed tree, then $(A, i)$ does not contain a finite permissible core. We know that in this case, the set of covolumes of non-uniform lattices in $\operatorname{Aut}(X), X=\widetilde{(A, i)}$, is not bounded away from zero, however our techniques do not suffice to produce a tower of groupings on $(A, i)$.

(4.2) Theorem. Let $(A, i)$ be as in Theorem (4.1). Let $X=\widetilde{(A, i)}$. Then there is an infinite ascending chain

$$
\Gamma_{1}<\Gamma_{2}<\Gamma_{3}<\cdots
$$

of non-uniform $X$-lattices in Aut $(X)$. Hence $\operatorname{Vol}\left(\Gamma_{i} \backslash \backslash X\right) \longrightarrow 0$ as $i \longrightarrow \infty$.

In Theorems (4.1), and (4.2), the covering tree $X=\widetilde{(A, i)}$ may be uniform or not. 


\section{Infinite towers of non-uniform $X$-lattices}

We have the following:

(5.1) Theorem $([\mathbf{R}])$. Let $(A, i)$ be a permissible edge-indexed graph. Suppose $(A, i)$ contains an arithmetic bridge with $n \geq 2$ edges. Then $(A, i)$ admits an infinite tower of finite faithful groupings.

Concerning existence of arithmetic bridges, we have the following:

(5.2) Theorem ([C1], [CR1]). Let $(A, i)$ be a unimodular edge-indexed graph. Let $e \in E A$ be a ramified edge such that $\Delta(e)$ is not an integer. If $e$ is not separating, then $e$ is contained in an arithmetic bridge with $n \geq 2$ edges.

Combining the results of $\S 2, \S 4$ and the above, we have:

(5.3) Theorem. Let $(A, i)$ be a permissible edge-indexed graph that is not a dominant-end-rooted edge-indexed tree. Then $(A, i)$ admits an infinite tower of finite faithful groupings.

A corollary of Theorem (5.3) is the following:

(5.4) Theorem. Let $X$ be a locally finite tree. If Aut $(X)$ contains a nonuniform $X$-lattice $\Gamma$, and $X$ is not the universal cover of a dominant-end-rooted edge-indexed tree, then Aut $(X)$ contains an infinite tower

$$
\Gamma_{1}<\Gamma_{2}<\Gamma_{3}<\cdots
$$

of non-uniform $X$-lattices. Hence $\operatorname{Vol}\left(\Gamma_{i} \backslash \backslash X\right) \longrightarrow 0$ as $i \longrightarrow \infty$.

\section{Existence of non-uniform $X$-lattices}

By Theorem (5.4), the question of existence of infinite towers of non-uniform $X$-lattices reduces to the question of existence of non-uniform $X$-lattices.

To outline the results on existence of non-uniform $X$-lattices, we make the following definition. Let $X$ be a locally finite tree, $G=\operatorname{Aut}(X)$, and let $\mu$ be a (left) Haar measure on $G$. Suppose that $G$ is unimodular. Then $\mu\left(G_{x}\right)$ is constant on $G$-orbits, so we can define ([BL], (1.5)):

$$
\mu(G \backslash \backslash X):=\sum_{x \in V(G \backslash X)} \frac{1}{\mu\left(G_{x}\right)} .
$$

We have the 'Lattice existence theorem':

(6.1) Theorem ([BCR], (0.2)). Let $X$ be a locally finite tree, let $G=\operatorname{Aut}(X)$, and let $\mu$ be a (left) Haar measure on $G$. The following conditions are equivalent:

(a) $G$ contains an $X$-lattice $\Gamma$.

(b) (U) $G$ is unimodular, and

(FV) $\mu(G \backslash \backslash X)<\infty$.

In particular, we have the following theorem, which together with Bass-Kulkarni's 'Uniform existence theorem' ([BK], (4.10)) gives Theorem (6.1): 
(6.2) Theorem ([BCR], (0.5)). Let $X$ be a locally finite tree, let $G=A u t(X)$, and let $\mu$ be a (left) Haar measure on $G$. Assume that:

(U) $G$ is unimodular,

(FV) $\quad \mu(G \backslash \backslash X)<\infty$, and

(INF) $G \backslash X$ is infinite.

Then $G$ contains a (necessarily non-uniform) $X$-lattice $\Gamma$.

For uniform trees, we have the following:

(6.3) Theorem ([C1], [C2]). If $X$ is uniform and not virtually rigid then $G$ contains a non-uniform $X$-lattice $\Gamma$.

\section{References}

[B] Bass, H, Covering theory for graphs of groups, J. Pure Appl. Algebra 89 (1993), 3-47.

[BCR] Bass, H, Carbone L, and Rosenberg, G, The existence theorem for tree lattices, Appendix [BCR], Tree Lattices by Hyman Bass and Alex Lubotzky (2000), Progress in Mathematics 176, Birkhauser, Boston.

[BK] Bass, H and Kulkarni, R, Uniform tree lattices, J. Amer. Math. Soc. 3 (4) (1990), 843-902.

[BL] Bass, H and Lubotzky, A, Tree lattices, Progress in Mathematics 176, Birkhauser, Boston (2000).

[BT] Bass, H and Tits, J, A discreteness criterion for certain tree automorphism groups, Appendix [BT], Tree Lattices by Hyman Bass and Alex Lubotzky (2000), Progress in Mathematics 176, Birkhauser, Boston.

[C1] Carbone, L, Non-uniform lattices on uniform trees, Mem. Amer. Math. Soc. 152 (July 2001, no. 724).

[C2] Carbone, L, Non-minimal tree actions and the existence of non-uniform tree lattices, Preprint (2001).

[CR1] Carbone, L and Rosenberg, G, Lattices on non-uniform trees, Submitted (2001).

[CR2] Carbone, L and Rosenberg, G, Infinite towers of non-uniform tree lattices, in preparation (2001).

[KM] Kazhdan, D and Margulis, G, A proof of Selberg's hypothesis. (Russian), Mat. Sb. (N.S.) 75 (117) (1968), 163-168.

[R] Rosenberg, G, Towers and covolumes of tree lattices, PhD. Thesis, Columbia University (2001).

[S] Serre, J.P, Trees (Translated from the French by John Stilwell) (1980), Springer-Verlag, Berlin Heidelberg.

Department of Mathematics, Harvard University, Science Center 325, One OxFord Street, CAmbridge, MA 02138.

E-mail address: lisa@math.harvard.edu

Department of Mathematics, Columbia University, New York, NY 10027.

E-mail address: gr@math.columbia.edu 